\title{
Joint Contact Forces can be Reduced by Improving Joint Moment Symmetry in Below-Knee Amputee Gait Simulations
}

\author{
Anne D. Koelewijn*, Antonie J. van den Bogert \\ Department of Mechanical Engineering, Cleveland State University, 1960 E 24th Str., \\ Cleveland, $\mathrm{OH} 44115$, USA
}

\begin{abstract}
Despite having a fully functional knee and hip in both legs, asymmetries in joint moments of the knee and hip are often seen in gait of persons with a unilateral transtibial amputation (TTA), possibly resulting in excessive joint loading. We hypothesize that persons with a TTA can walk with more symmetric joint moments at the cost of increased effort or abnormal kinematics. The hypothesis was tested using predictive simulations of gait. Open loop controls of one gait cycle were found by solving an optimization problem that minimizes a combination of walking effort and tracking error in joint angles, ground reaction force and gait cycle duration. A second objective was added to penalize joint moment asymmetry, creating a multi-objective optimization problem. A Pareto front was constructed by changing the weights of the objectives and three solutions were analyzed to study the effect of increasing joint moment symmetry. When the optimization placed more weight on moment symmetry, walking effort increased and kinematics became less normal, confirming the hypothesis. TTA gait improved with a moderate increase in joint moment symmetry. At a small cost of effort and abnormal kinematics, the peak hip extension moment in the intact leg was decreased significantly, and so was the joint contact force in the knee and hip. Additional symmetry required a significant increase in walking
\end{abstract}

\footnotetext{
* Corresponding author

Email addresses: a.koelewijn@csuohio.edu (Anne D. Koelewijn ),
} a.vandenbogert@csuohio.edu (Antonie J. van den Bogert)

Preprint submitted to Gait and Posture

June 24, 2016

(C) 2016. This manuscript version is made available under the Elsevier user license http://www.elsevier.com/open-access/userlicense/1.0/ 
effort and the joint contact forces in both hips became significantly higher than in able-bodied gait.

Keywords: Biomechanics, Simulation, Human Locomotion, Below-knee Amputee, Optimal Control

1

2

\section{Introduction}

The prevalence of limb amputation is expected to rise to one in 95 Americans in 2050. Most amputations are transtibial amputations due to complications of diabetes. However, amputations due to trauma are more common in young and physically active individuals (1). Persons with a transtibial amputation (TTA) who have worn a prosthesis for over five years have a higher occurrence rate of osteoarthritis in the knee and hip of the intact leg (2; 3). Also, other health issues exist among persons with an amputation, such as residual limb pain, back pain and phantom pain (4).

Studies analyzing gait of persons with a TTA have reported asymmetries between the residual and intact leg in the joint moments, the stance and swing time, the ground reaction force (GRF) (5) and EMG data (6). These asymmetries, especially in the joint loading, may cause higher forces in the intact leg compared to the residual leg and to able-bodied gait, which is suspected to be related to the aforementioned higher occurrence rate of osteoarthritis (2). Therefore, it is desirable to reduce the level of asymmetry in the joint moments 
of the knee and hip in TTA gait and distribute the load more equally between both legs.

The asymmetries are presumably caused by compensations required after amputation and the resulting loss of the biological ankle function (7) 8). Elastic storage and return (ESAR) feet were developed to improve TTA gait (9; 10). However, the joint moments remain distinctly asymmetric when using the ESAR feet $(11 ; 12,8)$.

Asymmetry is inevitable because the prosthetic limb does not replicate the mechanics and control of the intact limb. However, the knee and hip, and the entire intact limb, still have a sufficient set of muscles under voluntary neural control for flexion and extension. Therefore, we hypothesize that persons with a TTA can walk with symmetric joint moments using a passive prosthesis, but prefer not to do so because it would require additional effort or abnormal kinematics.

If humans choose their gait to minimize energy (13), this question can be investigated by predictive simulations of gait. In such simulations, an optimal control problem is formulated to find open loop controls that produce walking with minimal cost. Previous work showed that the main features of gait can be predicted by minimizing muscle activation (14, 15), and that predictions are improved when tracking of joint angles, GRFs and duration of normal gait is added to the objective (16). This objective has been used to predict the effect of sports equipment on performance (16) which is conceptually similar to predicting TTA gait, because both cases predict how an altered mechanical environment affects gait.

In the present study, a second objective is added to penalize asymmetry in joint moments. The second objective creates a multi-objective optimization, which is used to study the effect of different levels of joint moment symmetry on TTA gait. 


\section{Methods}

46 2.1. Model

47

48

73 $\mathrm{N} / \mathrm{m}, y_{0}=1 \cdot 10^{-3} \mathrm{~m}$. 
The horizontal GRF was modeled as a continuous approximation of Coulomb friction:

$$
F_{x}\left(f_{y}, \dot{x}\right)=-\mu F_{y} \frac{\dot{x}}{\sqrt{\dot{x}^{2}+v_{0}^{2}}}
$$

where $\dot{x}$ is the sliding velocity of the contact point, $\mu=1$ is the friction coefficient and $v_{0}=0.1 \mathrm{~m} / \mathrm{s}$ is a small velocity parameter that ensures a sign change in the friction when there is a change in the sliding direction.

Van den Bogert et al. (16) derived the dynamics equations for the multibody system with the ground contact model using Autolev (Online Dynamics, Sunnyvale, CA) with respect to the nine generalized coordinates $q$ :

$$
M(q) \cdot \ddot{q}+B(q, \dot{q})-\tau=0
$$

$M(q)$ is the mass matrix and $B(q, \dot{q})$ a vector that contains the Coriolis and gravity forces, the GRFs, and passive joint torques. The first three elements of the generalized force vector, $\tau$, are zero since these are unactuated degrees of freedom. The six joint moments, $\tau_{4}$ to $\tau_{9}$, are related to the muscle forces via the muscle moment arms, $d_{i j}$ using equation 5

$$
\left[\begin{array}{c}
\tau_{4} \\
\vdots \\
\tau_{9}
\end{array}\right]=\left[\begin{array}{cccc}
d_{1,1} & d_{1,2} & \ldots & d_{1,16} \\
\vdots & & \ddots & \vdots \\
d_{9,1} & d_{9,2} & \ldots & d_{9,16}
\end{array}\right] \cdot \bar{F}
$$

Muscle dynamics were formulated implicitly through the force balance equation (19):

$$
F_{S E E}\left(l_{C E}\right)=a F_{\text {max }} f\left(l_{C E}\right) g\left(i_{C E}\right)+F_{P E E}\left(l_{C E}\right)
$$

Where $F_{S E E}$ is the force in the series elastic element, $l_{C E}$ is the muscle fiber length, $a$ is the activation state, $F_{\max }$ is the maximal isometric force, $f$ and $g$ are the normalized force-length and force-velocity relationships, and $F_{P E E}$ is the force in the nonlinear parallel elastic element. Activation was controlled by neural controls $u$, bounded between zero and one, through an activation dynamics model. Details can be found in (19).

The multibody dynamics equations 4 and 5 were combined with the muscle dynamics, equation 6, and the ground contract model to form a set of implicit 
differential equations, $f(x, \dot{x}, u)=0$, with $x=\left[q, \dot{q}, l_{C E}, a\right]^{T}$. This function was coded in $\mathrm{C}$ and compiled as a MEX-function for MATLAB (Mathworks, Natick, MA). The partial derivatives of the function with respect to $x, \dot{x}$ and $u$ were also calculated by the function, as required for gradient-based optimization (19).

\subsection{Optimal Control Problem}

Predictive simulations were performed by solving an optimal control problem: find periodic state trajectories $x(t)$ and control trajectories $u(t)$ that satisfy the system dynamics and minimize a cost function. The formulation and solution method were identical to (16), but no left-right symmetry was assumed and a full gait cycle was simulated. A two-part cost function was used, using a weight $w$ to determine the relative importance of each objective:

$$
\min _{\bar{x}, \bar{u}} F_{1}(\bar{x}, \bar{u})+w \cdot F_{2}(\bar{x}, \bar{u})
$$

The first objective, $F_{1}$, was used by van den Bogert et al. (16) to perform predictive simulations of walking and running. This objective penalizes muscular effort and deviation from normal joint angles, GRFs and gait duration:

$$
\begin{aligned}
F_{1}(\bar{x}, \bar{u})= & \frac{1}{11}\left(\frac{1}{T} \sum_{k=1}^{10} \int_{0}^{T}\left(\frac{s_{k}(\bar{x}(t))-x_{m_{k}}(t)}{\bar{\sigma}_{k}}\right)^{2} \mathrm{~d} t\right. \\
& \left.+\left(\frac{T-T_{m}}{\sigma_{T}}\right)^{2}\right)+\frac{W_{\text {effort }}}{16 T} \sum_{j=1}^{16} \int_{0}^{T} u_{j}(t)^{2} \mathrm{~d} t
\end{aligned}
$$

The eleven tracked variables $s_{k}(\bar{x}(t))$ were six joint angles, the horizontal and vertical GRFs of both legs, and the duration of the gait cycle, T. Tracking data, $x_{m_{k}}(t)$ and $T_{m}$, and standard deviations, $\bar{\sigma}_{k}$, were provided by Winter (20). Based on (16), $W_{\text {effort }}=10$ was used to scale the effort term.

The second objective function, $F_{2}$, was added to study the effect of encouraging symmetric joint moments in knee and hip. Joint moment symmetry is encouraged indirectly by the tracking term in $F_{1}$, but the amputation makes the model asymmetrical. Asymmetrical movement will therefore occur if this reduces the effort term in $F_{1}$. Furthermore, even if joint angles and GRFs are symmetric, there may be asymmetry in the center of pressure, which affects joint 
moments but is not included in the tracking term. Joint moment asymmetry was defined as the root mean square difference between the left and right joint moment, accounting for a phase shift of a half-cycle:

$$
F_{2}(\bar{x}, \bar{u})=\frac{1}{2} \sum_{i=1}^{2} \int_{0}^{T}\left(M_{i_{r}}(t)-M_{i_{l}}\left(t+\frac{T}{2}\right)\right)^{2} \mathrm{~d} t
$$

The optimal control problem was solved through direct collocation, with 60 collocation nodes per gait cycle and a backward Euler formulation. One node was added to ensure periodicity of the gait cycle, so the final state matches the initial state with a forward translation. Details can be found in $(\underline{15})$ and $(\underline{16})$. The resulting constrained optimization problem was solved using IPOPT 3.11.0 (21).

First, the optimal control problem was solved for the able-bodied model (ABLE). To obtain this solution, standing was solved first, followed by a series of able-bodied gait solutions with increasing speed. Then, using the able-bodied solution as an initial guess, TTA gait was solved using only objective $F_{1}$, thus without considering asymmetry in joint moments (TTA1).

Then, objective $F_{2}$ was added, creating a multi-objective optimization problem for TTA gait due to the asymmetry between the left and right leg. A Pareto-front was obtained by solving the optimization problem using different weights for objective $F_{2}$.

Two solutions (TTA2 and TTA3) on the Pareto-front were analyzed and compared against the baseline and the able-bodied solution to analyze the effect of $F_{2}$. Joint angles, joint moments, muscle forces, GRFs and joint contact forces were examined to find kinetic and kinematic changes. The energy required for each of the predicted gaits is compared using the effort term in equation 8 and a metabolic cost model (22). GRFs, joint angles and muscle forces were used to find the joint contact force (JCF) magnitudes, the Euclidean norm of the components in the $\mathrm{x}$ - and $\mathrm{y}$-direction. 


\section{Results}

First, able-bodied gait was solved. A gait cycle of 1.14 seconds with a speed of $1.325 \mathrm{~m} / \mathrm{s}$ required $3.12 \mathrm{~W} / \mathrm{kg}$ metabolic energy. Joint angles and ground reaction forces of this solution were all within one standard deviation of the tracking data (20). Only the peak hip flexion angle was slightly higher. The able-bodied solution was found in 7 minutes and 52 seconds on a computer with an Intel Core i5-3210M CPU at $2.5 \mathrm{GHz}$ clock speed.

The Pareto-front (Figure 2) shows the trade-off between symmetry in the joint moment and the objective of low effort and normal gait. Initially, the increase in joint moment symmetry had a small effect on the tracking and effort term. However, as joint moment symmetry improved, the cost of more joint moment symmetry increased considerably. All gaits are theoretically feasible for a person with a TTA, the choice between them depending on the relative weight of the optimization objectives, which are reported in figure 3

Three points are of interest in the Pareto-front. Solution TTA1 is the baseline solution, where the weight of objective $F_{2}$ was zero. Solution TTA2, which used $w=0.3$, is a solution where the symmetry of the joint moments greatly improved with little extra effort and deviation from normal kinematics (11\% increase in F1). Solution TTA3, where $w=10$, required an increase in effort and tracking error of almost $33 \%$.

Figure 3 shows the tracking error, effort, RMS moment asymmetry and metabolic cost as a function of the weight $w$ in equation 7 . Tracking error increased almost linearly from 0.55 at $w=0$ to 0.79 at $w=10$. Effort stayed approximately constant at 0.0257 until $w=0.2$, after which it increased to 0.0282 at TTA3. Joint moment asymmetry decreased from $17.5 \mathrm{Nm}$ to $5.19 \mathrm{Nm}$ at $w=0.2$ and then to $0.635 \mathrm{Nm}$ at solution TTA3. The metabolic cost first decreased from $3.09 \mathrm{~W} / \mathrm{kg}$ to $3.05 \mathrm{~W} / \mathrm{kg}$ at $w=0.05$, after which it increases to $3.21 \mathrm{~W} / \mathrm{kg}$ at TTA3.

Joint angles (Figure 4) were within one standard deviation of the tracking data, except the peak hip flexion angle at heel strike, which deviated more 
than two standard deviations, and the intact knee angle in TTA3 between $20 \%$ and $40 \%$ of the gait cycle, which deviated more than one standard deviation. Furthermore, the knee and hip joint kinematics were similar and nearly symmetric in all solutions. However, the joint kinetics distinctly differed between the solutions.

In TTA 1, which did not consider joint moment asymmetry, joint moments were distinctly asymmetric and differed from ABLE (Figure 4). The peak extension moment in the hip of the prosthesis side was $38 \mathrm{Nm}$, compared to 100 $\mathrm{Nm}$ on the intact side and $44 \mathrm{Nm}$ in ABLE. The peak extension moment in the knee was $28 \mathrm{Nm}$ on the prosthesis side and $51 \mathrm{Nm}$ in the intact side, compared to $42 \mathrm{Nm}$ in ABLE.

In solution TTA2, tracking error increased by $16 \%$, effort increased by $2 \%$ and joint moment asymmetry was reduced by $76 \%$. Peak extension moment in the hip was $67 \mathrm{Nm}$ on the prosthesis side and $76 \mathrm{Nm}$ on the intact side, more symmetrical than in TTA1 but higher than in ABLE. The peak knee extension moment was also more symmetrical, $26 \mathrm{Nm}$ on the prosthesis side and $34 \mathrm{Nm}$ on the intact side. Both values were lower than in ABLE.

Solution TTA3 reduced joint moment asymmetry by $88 \%$, but required an increase in effort of $10 \%$ and in tracking error of $43 \%$. In this solution, the peak hip extension moment was equal to $89 \mathrm{Nm}$ on the prosthesis side and $90 \mathrm{Nm}$ on the intact side, more than twice as high as in ABLE. The peak knee moment is equal to $22 \mathrm{Nm}$ on the prosthesis side and $23 \mathrm{Nm}$ on the intact side, which is about half that of ABLE.

Figure 5 shows the muscle forces in the musculoskeletal model. Hip muscle forces were lowest in ABLE. In TTA1, the Hamstrings force was high in early stance on the intact side, $603 \mathrm{~N}$, compared to $356 \mathrm{~N}$ on the prosthesis side. The peak force in the Gluteals was $986 \mathrm{~N}$ on the intact side, compared to 307 $\mathrm{N}$ on the prosthesis side. Also, the peak force in the Iliopsoas was high on the prosthesis side during late stance, $883 \mathrm{~N}$, compared to $280 \mathrm{~N}$ on the intact side. In TTA2 and TTA3, peak forces in the hip muscles on the prosthesis side increased compared to TTA1. The peak force in the Gluteals on the intact side 
decreased, and so did the peak force in the Hamstrings in TTA2. The other peak forces remained similar to TTA1.

The vertical GRF on the prosthesis side was similar for all solutions and within one standard deviation of the tracking data (Figure6). Large differences were seen in the JCFs (Figure 6). Hip JCFs were higher than in ABLE for both legs in all conditions. In the intact side, the peak JCF was lowest in TTA2, but higher than in ABLE. The JCF in the knee of the prosthesis side was lower than ABLE for all solutions.

\section{Discussion}

An able-bodied solution was found using the approach presented by van den Bogert et al. (16). Joint moments are symmetric between the two legs. Their values correspond to results found in studies with human subjects. Also, the metabolic energy was similar to metabolic energy found in studies with human subjects (23).

Any of the solutions on the Pareto-front can theoretically be chosen as a gait by persons with a TTA. Therefore, the hypothesis that persons with a TTA can walk with more symmetry in the joint moments at the cost of increased effort and abnormal kinematics is confirmed. Figure 3 shows that joint moment asymmetry can be reduced by $70 \%$ with little increase in effort or metabolic cost.

If persons with a TTA select a gait with normal kinematics and minimal effort, we would expect them to select solution TTA1. The largely symmetric joint angles in the knee and hip in TTA1 are also seen in studies of persons with a TTA $(11 ; 24)$. The predicted reduction of the knee joint moment on the prosthesis side in TTA1 is also seen in subjects with a TTA (8; 11; 12).

Nolan and Lees (25) and Ferris et al. (8) report a higher peak extension hip moment in the intact leg compared to able bodied walking, which agrees with our predictions in TTA1. However, some literature reports more symmetry in the peak hip extension moment of the intact and residual leg $(26$; 27), which is 
more similar to TTA2. TTA1 and TTA2 require a similar amount of effort or metabolic work, which suggests persons with a TTA operate around TTA1 and TTA2 in the Pareto-front.

Solution TTA2 has several advantages over TTA1 and TTA3. A lower peak extension moment in the hip and knee reduced joint moment asymmetry and yielded a lower JCF in both joints. A high JCF is suspected to be related to a higher incidence rate of osteoarthritis in persons with a TTA (2), so the lower JCFs could decrease this higher incidence rate. Another potential benefit in TTA2 is the higher knee JCF in the residual leg compared to TTA1 and to ABLE, which might reduce the loss of bone mineral density in the proximal tibia and femur neck, which is reported for persons with a TTA (28). Therefore, teaching persons with a TTA to walk using TTA2 may be useful in rehabilitation.

TTA3 emphasized joint moment symmetry too much, which is disadvantageous for TTA gait. Apart from a 10\% increase in effort compared to TTA1, the peak hip extension moment increased in both legs, which also yielded a higher JCF in the hip. Currently, prevalence of osteoarthritis in the intact hip is already higher in persons with a TTA (3) and TTA3 could increase the risk of developing osteoarthritis in the hips of both legs.

On the prosthesis side, there is no active push-off and the biarticular Gastrocnemius is removed completely. This might cause the Iliopsoas force to increase on the prosthesis side during late stance, because this muscle now initiates the swing phase and compensates for the activation of the biarticular Hamstrings, which flexes the knee instead of the Gastrocnemius. Some persons with a TTA can still use the Gastrocnemius to control the knee, which might reduce this effect. Additionally, the lack of push-off increases the impact force on the intact side (Figure 6) (29). A passive foot that stores and releases energy using microprocessor control, such as the artifical foot by Collins and Kuo (29) or a prosthesis with a motor can better restore the push-off function of the biological ankle and reduce these effects.

The metabolic model produced a lower metabolic cost for TTA gait than 
for able-bodied gait, which contradicts subject studies (23). The predicted metabolic cost for TTA gait is lower because three muscles are removed that would otherwise contribute significantly. While our predictions are theoretically possible, actual TTA gait may require more energy due to antagonistic cocontraction to stabilize the prosthetic side (6), for example due to an unstable connection between the prosthesis and residual leg. Such co-contraction will not be predicted by a simulation where effort is optimized and no stability is required. Therefore, we conclude that other objectives might also influence TTA gait, like stability, joint pain or comfort. The underestimation of metabolic energy suggest that we may also have underestimated the muscle forces and JCFs.

The modeled prosthesis had the same mass and moment of inertia as the intact leg. This ensured that this study only predicted effects due of control and not of a change in prosthesis parameters. Prosthetic legs have different mass and inertial properties than a normal leg, but this will mostly affect the swing phase, where forces and moments are low. Therefore, we expect that there is little effect of mass properties on the peak loading variables.

This study used a two-dimensional musculoskeletal model. Hip joint forces may have been underestimated by neglecting the abductor moments. However, we expect the main conclusions to be valid. This should be verified with a three dimensional musculoskeletal model or with subject tests. Further tests with a three dimensional model could also analyze the effects of having joint moment symmetry in the frontal and transverse plane, while a subject study could verify if subjects can maintain the suggested gait modifications.

We conclude from this study that:

1. Asymmetric knee and hip joint moments in persons with a TTA with a passive foot can be explained by a predictive simulation model that minimizes effort and deviation from normal kinematics.

2. Considerable improvement in moment symmetry is theoretically possible with a small increase in effort and almost no change in kinematics. The 
hip joint moment will, however, remain elevated above able-bodied values, which is currently also reported for persons with a TTA $(26,27)$.

\section{Acknowledgments}

This research was supported by the National Science Foundation under Grant No. 1344954 and by a Graduate Scholarship from the Parker-Hannifin Corporation.

\section{Conflict of Interest Statement}

There are no conflicts of interest for any of the authors regarding the research reported in this manuscript.

\section{References}

[1] K. Ziegler-Graham, E. J. MacKenzie, P. L. Ephraim, T. G. Travison, R. Brookmeyer, Estimating the prevalence of limb loss in the united states: 2005 to 2050, Archives of Physical Medicine and Rehabilitation 89 (3) (2008) 422-429.

[2] E. D. Lemaire, F. R. Fisher, Osteoarthritis and elderly amputee gait, Archives of Physical Medicine and Rehabilitation 75 (1994) 1094-1099.

[3] P. A. Struyf, C. M. van Heugten, M. W. Hitters, R. J. Smeets, The prevalence of osteoarthritis of the intact hip and knee among traumatic leg amputees, Archives of physical medicine and rehabilitation 90 (3) (2009) 440446.

[4] P. L. Ephraim, S. T. Wegener, E. J. MacKenzie, T. R. Dillingham, L. E. Pezzin, Phantom pain, residual limb pain, and back pain in amputees: results of a national survey, Archives of Physical Medicine and Rehabilitation 86 (10) (2005) 1910-1919. 
[5] M. R. Menard, M. E. McBride, D. J. Sanderson, D. D. Murray, Comparative biomechanical analysis of energy-storing prosthetic feet, Archives of Physical Medicine and Rehabilitation 73 (5) (1992) 451-458.

[6] E. Isakov, O. Keren, N. Benjuya, Trans-tibial amputee gait: Time-distance parameters and emg activity, Prosthetics and Orthotics International 24 (3) (2000) 216-220.

[7] G. Hurley, R. McKenney, M. Robinson, M. Zadravec, M. Pierrynowski, The role of the contralateral limb in below-knee amputee gait, Prosthetics and Orthotics International 14 (1) (1990) 33-42.

[8] A. E. Ferris, J. M. Aldridge, C. A. Rábago, J. M. Wilken, Evaluation of a powered ankle-foot prosthetic system during walking, Archives of Physical Medicine and Rehabilitation 93 (11) (2012) 1911-1918.

[9] N. P. Fey, G. K. Klute, R. R. Neptune, The influence of energy storage and return foot stiffness on walking mechanics and muscle activity in belowknee amputees, Clinical Biomechanics 26 (10) (2011) 1025-1032.

[10] J. Wagner, S. Sienko, T. Supan, D. Barth, Motion analysis of sach vs. flex-foot in moderately active below-knee amputees, Clinical Prosthetics \& Orthotics 11 (1) (1987) 55-62.

[11] D. J. Sanderson, P. E. Martin, Lower extremity kinematic and kinetic adaptations in unilateral below-knee amputees during walking, Gait and Posture 6 (2) (1997) 126-136.

[12] H. Bateni, S. J. Olney, Kinematic and kinetic variations of below-knee amputee gait, JPO: Journal of Prosthetics and Orthotics 14 (1) (2002) $2-10$.

[13] J. E. Bertram, A. Ruina, Multiple walking speed-frequency relations are predicted by constrained optimization, Journal of Theoretical Biology 209 (4) (2001) 445-453. 
[14] F. C. Anderson, M. G. Pandy, Dynamic optimization of human walking, Journal of Biomechanical Engineering 123 (5) (2001) 381-390.

[15] M. Ackermann, A. J. van den Bogert, Optimality principles for model-based prediction of human gait, Journal of Biomechanics 43 (6) (2010) 1055-1060.

[16] A. J. Van den Bogert, M. Hupperets, H. Schlarb, B. Krabbe, Predictive musculoskeletal simulation using optimal control: effects of added limb mass on energy cost and kinematics of walking and running, Proceedings of the Institution of Mechanical Engineers, Part P: Journal of Sports Engineering and Technology (2012) 1-11.

[17] D. A. Winter, Biomechanics and motor control of human movement, John Wiley \& Sons, 2005.

[18] R. J. Zmitrewicz, R. R. Neptune, K. Sasaki, Mechanical energetic contributions from individual muscles and elastic prosthetic feet during symmetric unilateral transtibial amputee walking: a theoretical study, Journal of Biomechanics 40 (8) (2007) 1824-1831.

[19] A. J. Van den Bogert, D. Blana, D. Heinrich, Implicit methods for efficient musculoskeletal simulation and optimal control, Procedia IUTAM 2 (2011) 297-316.

[20] D. A. Winter, Biomechanics and motor control of human gait: normal, elderly and pathological, University of Waterloo Press, 1991.

[21] A. Wächter, L. T. Biegler, On the implementation of an interior-point filter line-search algorithm for large-scale nonlinear programming, Mathematical Programming 106 (1) (2006) 25-57.

[22] B. R. Umberger, K. G. Gerritsen, P. E. Martin, A model of human muscle energy expenditure, Computer methods in biomechanics and biomedical engineering 6 (2) (2003) 99-111. 
[23] D. Hunter, E. S. Cole, J. M. Murray, T. D. Murray, Energy expenditure of below-knee amputees during harness-supported treadmill ambulation, Journal of Orthopaedic \& Sports Physical Therapy 21 (5) (1995) 268-276.

[24] E. Isakov, H. Burger, J. Krajnik, M. Gregoric, C. Marincek, Influence of speed on gait parameters and on symmetry in transtibial amputees, Prosthetics and Orthotics International 20 (3) (1996) 153-158.

[25] L. Nolan, A. Lees, The functional demands on the intact limb during walking for active trans-femoral and trans-tibial amputees, Prosthetics and Orthotics International 24 (2) (2000) 117-125.

[26] N. Vanicek, S. Strike, L. McNaughton, R. Polman, Gait patterns in transtibial amputee fallers vs. non-fallers: Biomechanical differences during level walking, Gait and Posture 29 (3) (2009) 415-420.

[27] J. D. Ventura, G. K. Klute, R. R. Neptune, The effects of prosthetic ankle dorsiflexion and energy return on below-knee amputee leg loading, Clinical Biomechanics 26 (3) (2011) 298-303.

[28] T. Royer, M. Koenig, Joint loading and bone mineral density in persons with unilateral, trans-tibial amputation, Clinical Biomechanics 20 (10) (2005) 1119-1125.

[29] S. H. Collins, A. D. Kuo, Recycling energy to restore impaired ankle function during human walking, PLoS one 5 (2) (2010) e9307.

[30] A. Gitter, J. M. Czerniecki, D. M. DeGroot, Biomechanical analysis of the influence of prosthetic feet on below-knee amputee walking., American Journal of Physical Medicine \& Rehabilitation 70 (3) (1991) 142-148. 


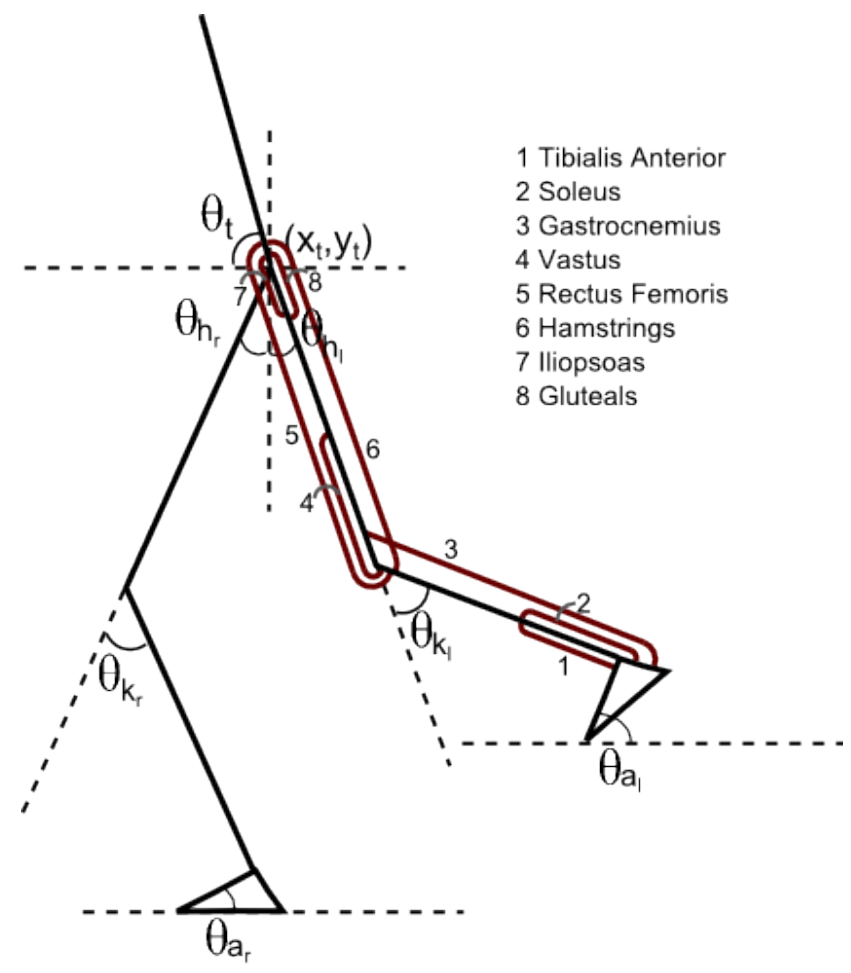

Figure 1: Sagittal plane model that is used in the predictive simulations.

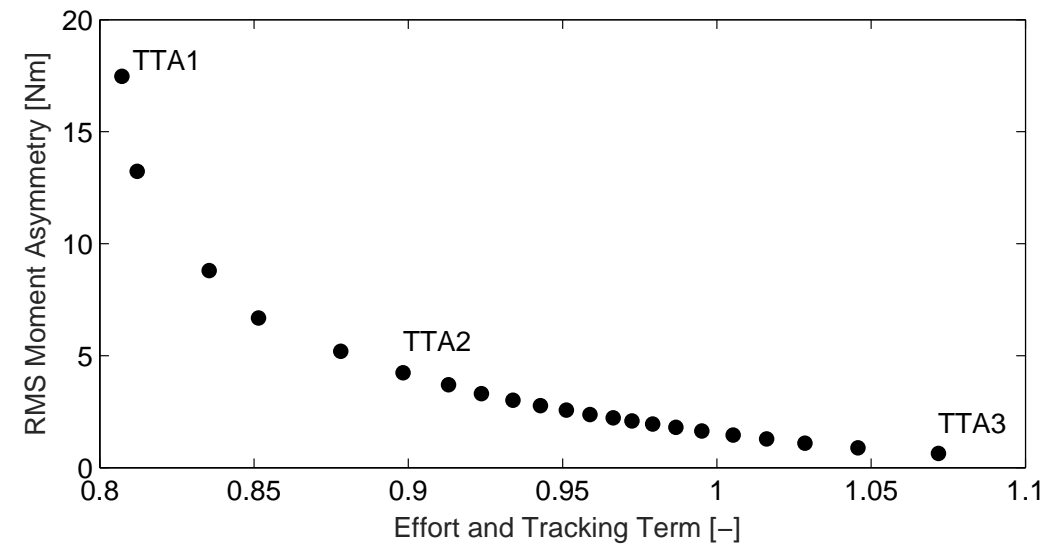

Figure 2: Pareto-front showing the trade-off between effort and symmetry in the joint moments. Solutions TTA1, TTA2 and TTA3 will be analysed further. 

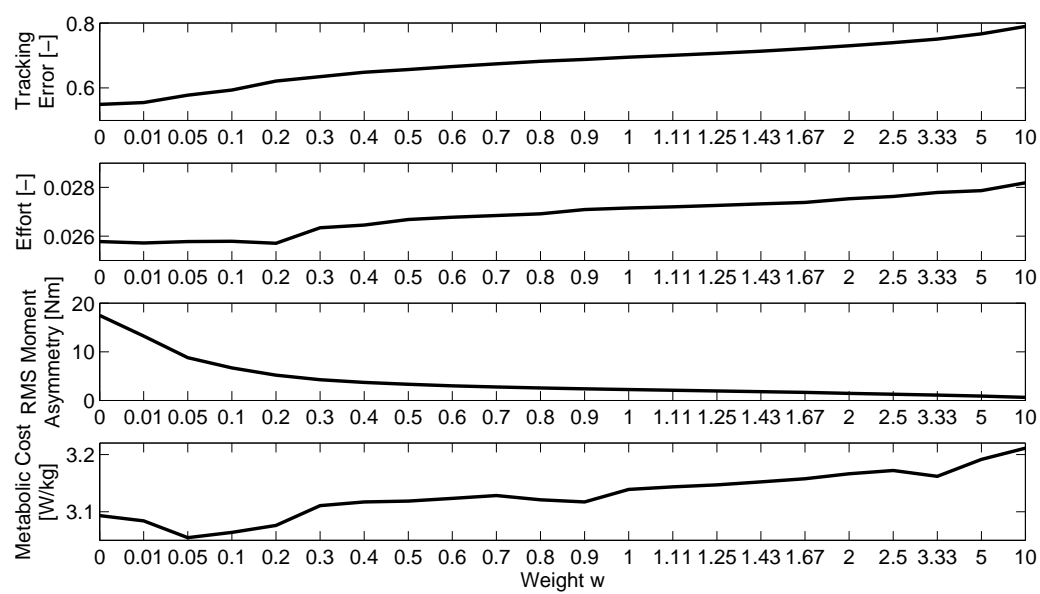

Figure 3: Figure showing the different objectives of tracking error, effort and moment symmetry, as well as the metabolic cost as a function of the weight $w$. 

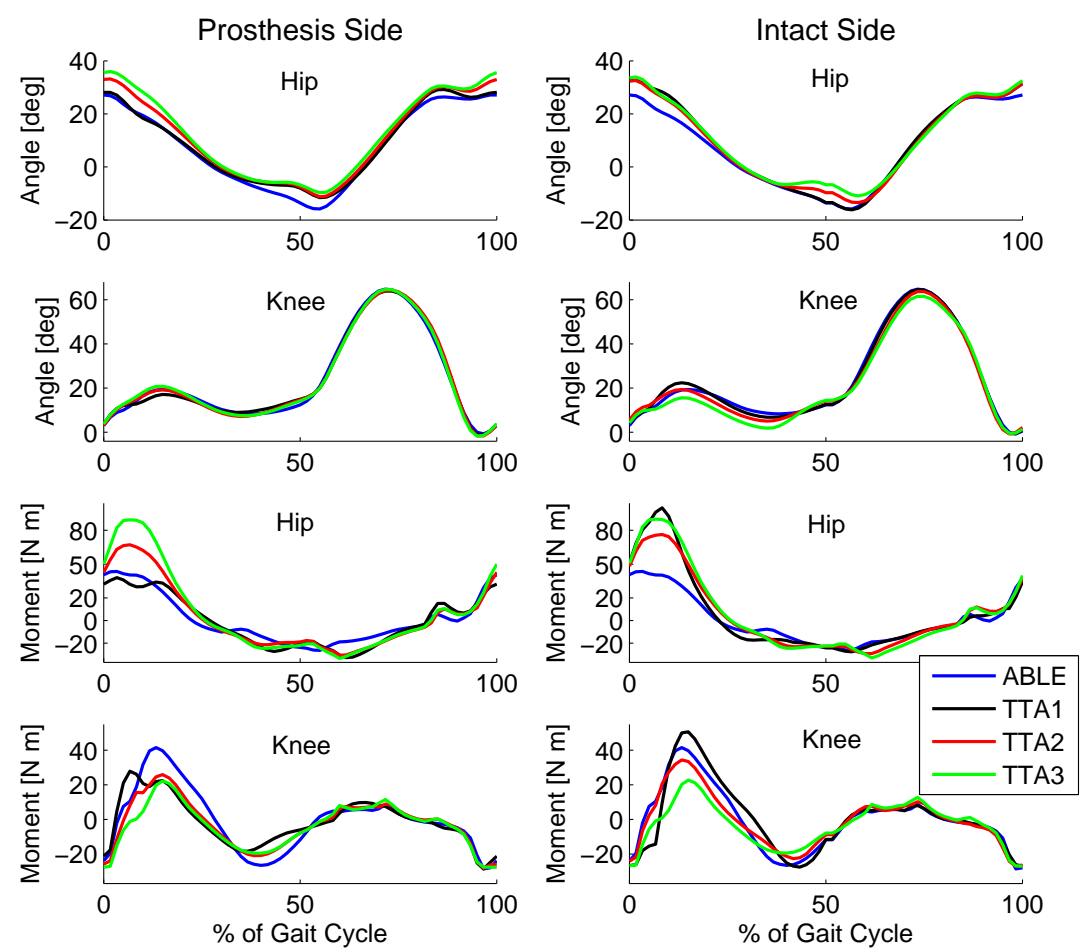

Figure 4: Joint angles, moments for the prosthesis side (left) and the intact side (right) for solutions ABLE, TTA1, TTA2 and TTA3. The gait cycle starts at heel strike on both sides. The angles show flexion positive, while in the moment graphs, extension is positive. 

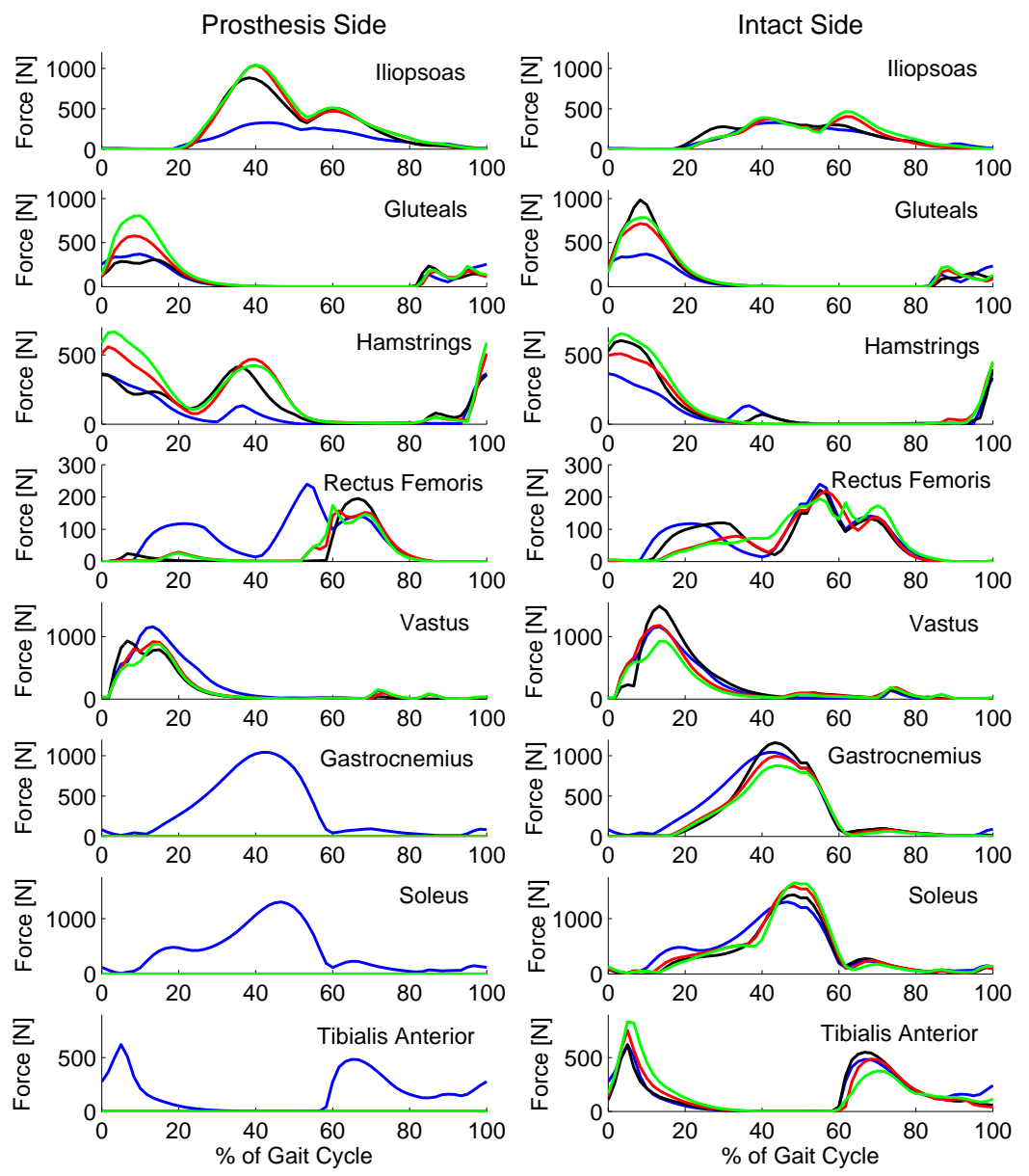

$\longrightarrow$ ABLE $\longrightarrow$ TTA1 - TTA2 TTA3

Figure 5: Muscle forces of all muscles in the musculoskeletal model for solutions ABLE, TTA1, TTA2 and TTA3. The gait cycle starts at heel strike on both the prosthesis side (left) and the intact side (ride). 
Prosthesis Side
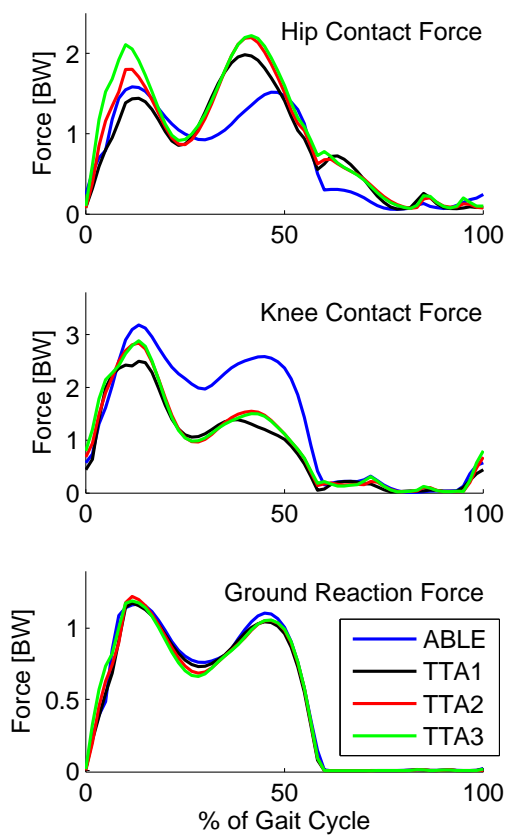

Intact Side
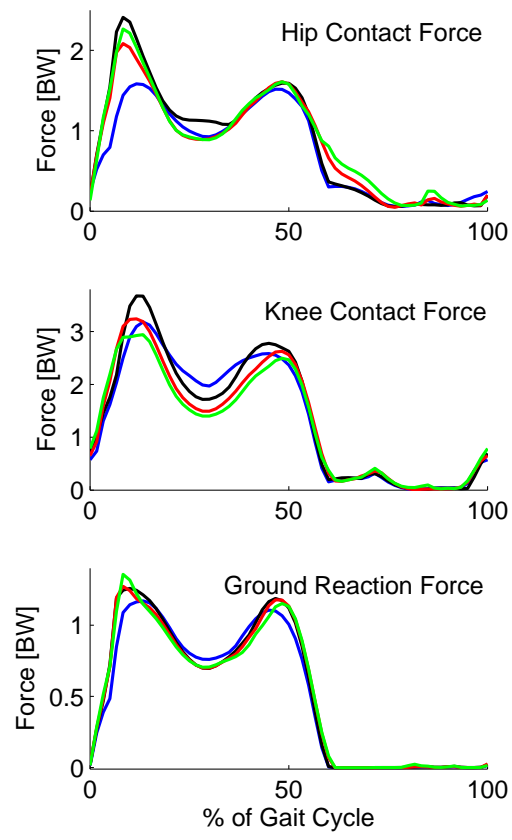

Figure 6: Joint contact force in the knee and hip for solutions ABLE, TTA1, TTA2 and TTA3. 